\title{
THE FUTURE OF THE ESOPHAGUS CANCER
}

Malafaia O. The future of the esophagus cancer. Arq Gastroenterol. 2013;50(2):79-80.

HEADINGS - Esophageal neoplasms.

Surgical treatment of esophageal cancer turns esophagologist into "sarcophagologist"!

The above striking phrase positions very well the surgical treatment of this cancer until the first half of last century. Historical accounts refer that these words were spoken currently in Americans and Europeans hospital corridors by that time. Although blunt, the phrase represented metaphorically the truth and, besides this, quick death sentence and miserable life to whom had the diagnosis. Little could be offered to the patients, not even death with dignity.

The description of the first report of esophageal cancer is attributed to White in $1898^{(1)}$ and the first esophagectomy - only cervical without reconstruction of digestive transit - to Theodor Billroth and Czerny in 1870. The thoracic esophagus was only successfully resected via the chest in 1913 by Philip Thorek, offering remarkable survival to the patient; however, the food transit was not reinstalled by him leaving permanent esophagostomy and gastrostomy, witch compromised the quality of life during the long survival of the operated patient ${ }^{(7,13)}$.

This was the way how esophageal cancer treatment began!

\section{Attempt to overcome mortality}

Decades followed in which the technique of choice for resection of esophageal cancer was that of Lewis ${ }^{(3)}$ and Tanner ${ }^{(12)}$. With it, the tumor and adjacent lymph nodes were resected using combined access with right thoracotomy with laparotomy. The reconstruction of the digestive tract was almost always carried out with the stomach through high intra-thoracic esophagogastric anastomosis. Mortality was high, up to $12 \%$ and morbidity, associated with, between $50 \%$ and $60 \%$. Survival was very short, considering what could be expected with the complexity and severity of the surgery ${ }^{(14)}$.

Hand in hand with this, there was an increased incidence of esophageal cancer since 70's, at the expense of adenocarcinoma of the esophagogastric junction associated with Barrett's esophagus. This was one of the reasons why this cancer was re-considered on its treatment, gaining more attention from surgeons looking for other alternative therapies with lower morbidity. Techniques were improved with the advent of mechanical sutures and other new surgical alternatives sought to minimize the surgical aggression. In this aspect, the contribution of Pinotti and his group in 1977 at the University of São Paulo, Brazil, with the transmediastinal esophagectomy and cervical esophagogastric anastomosis - removing it from the inside of the chest -, was remarkable ${ }^{(4,5,6)}$. This operation initially was applied to the treatment of advanced achalasia and, subsequently, to esophageal cancer. At the same time, significant advances on patient's care with postoperative intensive treatment in specific units, began to be more offered and with better quality. Also, clinical therapy and nutritional support in extensive operations contributed to change the dark specter hanging over these patients.

All these factors together, in fact, resulted in sharp decline in mortality. Exorbitant morbidity of $60 \%$ on the past, also deeply plummeted.

\section{Attempts to increase survival}

Passed the phase of avoiding death by the surgical procedure, the present time is concerned with increasing disease-free survival.

To diminishes the locoregional recurrences that continue to occur after operations, were proposed procedures with extended lymphadenectomy, i.e., esophagectomy with lymphadenectomy in three fields - abdominal, mediastinal and cervical ${ }^{(2,11)}$ - or radical mediastinal lymphadenectomy of lymph nodes and structures of the posterior mediastinum ${ }^{(8,10)}$. These procedures are encouraged by the Japanese school. However, the results obtained there have not been achieved in the West. It seems that the tolerance to human aggression is overtaken by the surgical procedure, in this side of the world. The long-term outcomes with controlled studies are still not available. Thus, caution should be used when using these extensive resections, perhaps selecting patients who may be benefited by it.

The chemo and radiation therapy, alone or associated in various combinations, have sought and achieved some results. Several chemotherapy drugs are giving less morbidity and greater comfort in treatment. However, the purpose to increase survival with therapeutic procedures as isolated treatment, i.e., not associated with surgery, has not been demonstrated forcefully. There are still a lot of controversies. Paper on neoadjuvant chemoradiotherapy associated with surgical treatment compared to surgery alone in squamous cell carcinoma of the esophagus in this issue of the ARCHIVES of GASTROENTEROLOGY, brings interesting information. Andreollo NA, et al. in a well-conducted study assessing the survival time in patients operated for squamous cell carcinoma of the esophagus with or without neoadjuvant chemoradiotherapy, concluded that the use of this method in patients with squamous cell carcinoma of the esophagus offers benefits and increase the survival.

Thoracoscopic surgical methods, minimally invasive techniques and laparoscopic access are still in early stage results. Signalize to be equivalent to the procedures con- 
ventionally used, but with less morbidity. There is no certainty as to further survival time, because long-term monitoring is not widely available and studies preferably include patients with less advanced stages of the disease, which can bring selection bias to the results. We have to wait.

But for superficial tumors exclusively situated in the mucosa, there are conditions to predict, in medium-term future time, minimally invasive resection done by endoscopy. The reader can also see this matter in another interesting paper also published in this issue of the ARCHIVES of GASTROENTEROLOGY. Under the title "Initial experience of endoscopic submucosal dissection in Brazil to treat early gastric and esophagheal cancer: a multi-institutional analysis", Chaves MD et al. evaluate the feasibility of the technique of endoscopic submucosal dissection in the treatment of 14 patients with squamous cell carcinoma of the esophagus; 13 were resected en-bloc and one of them developed esophageal stricture. The mean follow-up reported is still short ( 8.6 months), but there was no local recurrence in this period. The authors conclude be technically feasible the endoscopic treatment with a high successful rate.

If these endoscopic procedures will continuously be successful for control and cure of the disease, also only time will tell. However, to avoid esophagectomy - a procedure of high complexity and great agression - is always desirable. Since in long follow-ups the recurrence rates demonstrate to be less than or equal the standard operation, minimally invasive means are the hope for tumors incidentally diagnosed in early stages.

With these concepts in mind, the treatment of esophageal cancer nowadays has changed its strategy. It should be focused on tailoring treatment to each patient, considering always the potentiality on survival and quality of life that therapeutic options may provide per $\mathrm{se}^{(9,15)}$.

And so... the surgeon is no longer a "sarcophagologist!"

\section{Perspectives}

Although with improvements, yet about $2 / 3$ of patients with esophageal cancer in the West have unresectable disease at diagnosis due to late demand for medical help. Thus, the extent of locoregional disease or distant metastasis are present at diagnosis. In addition, patients may not be candidates for more extensive operations due to medical comorbidities often associated.

The various combinations of cytotoxic agents and periodicities infusion contributed to extend the disease-free interval, but the impact on survival remains below the desired level ${ }^{(14)}$. The same applies to radiotherapy methods.

Nowadays, surgery offers the only chance of cure for patients with localized disease and in early stages. However, the surgical success rate is compromised by the high incidence of the disease in high level staging process and with advanced tumors with local and systemic recurrences; these circumstances decrease survival at 5 years.

It seems that we need a new way, different from the present.

Thus, biological therapy, which has the paradigm to resemble the organic reactions to combat diseases - including cancer - is very promising for the future in the medium term. Although still in its infancy, authorized and controlled closed studies using several substances intent to increase disease-free survival. Preliminary results of these studies are very promising indicating possible new path - so desire by doctors. Only time will tell if it is feasible and better than current methods, which appear to be near to exhaustion.

Studies on better understanding the carcinogenesis, genomic instability and the development of genetic engineering to correct the genetic defect that leads to tumor, may be defined in the future, but... in the very long time! Then, yes: there will be a radical therapeutic change, different from what we have today, and perhaps the cure finally can be reached!

Before this happens, it is likely that the adoption of screening programs for early diagnosis and public awareness of risks and preventive measures will help to bring to medical assistance patients in better disease stages, and thereby creating to themselves better opportunity of have longer survival.

\section{"Nowadays, in relationship to esophageal cancer and its treatment, if we are not sailing under blue sky, at least we can see the sunshine among the clouds"}

\section{Osvaldo MALAFAIA*}

\section{REFERENCES}

1. DeMeester SR. Adenocarcinoma of the esophagus and cardia: a review of the disease and its treatment. Ann Surg Oncol. 2005;13:12-30.

2. Lerut T, Nafteux P, Moons J, Coosemans W, Decker G, De Leyn P Three-field lymphadenectomy for carcinoma of the esophagus and gastroesophageal junction in 1974 R0 ressections: impact on staging, disease-free survival, and outcome. Ann Surg. 2004;240:962-74.

3. Lewis I. The surgical treatment of carcinoma of the esophagus with special reference to a new operation for growths of the middle third. Br J Surg. 1946:34:18-31.

4. Pinotti HW. Esofagectomia subtotal, por túnel transmediastinal sem toractomia. Rev Assoc Med Bras. 1977;23:395-8

5. Pinotti HW, Zilberstein B, Pollara W, Raia A. Esophagectomy without thoracotomy. Surg Gynecol Obstet. 1981;152:344-6.

6. Pinotti HW, Cecconello I, Oliveira MA. Transhiatal esophagectomy for esophageal cancer. Semin Surg Oncol. 1997;13(4):253-8.

7. Pompilli MF, Mark JBD. The history of surgery for carcinoma of the esophagus. Chest Surg Clin N Am. 2000;10:145-51.

8. Skinner DB. En bloc resection for neoplasms of the esophagus and cardia. J Thorac Cardiovasc Surg. 1983;85:59-71.

9. Stein HJ, Siewert JR. Improved prognosis of resected esophageal cancer. World J Surg. 2004:28:520-5.

10. Swanson SJ, Batirel HF, Bueno R, Jaklitsch MT, Lukanich JM, Allred E. Transthoracic esophagectomy with radical mediastinal and abdominal lymph node dissection and cervical esophagogastrostomy for esophageal carcinoma. Ann Thorac Surg. 2001;72:1918-25.

11. Tachibana M, Kinugasa S, Yoshimura H, Shibakita M, Tonomoto Y, Dhar DK. Clinical outcomes of extended esophagectomy with three-field lymph node dissection for esophageal squamous cell carcinoma. Am J Surg. 2005;189:98-109.

12. Tanner NC. The present position of carcinoma of the esophagus. Postgrad Med J. 1947;23:109-39.

13. Thorek P. The operative treatment of carcinoma of the esophagus. Ann Surg. 1915;61:385-405.

14. Tytgat GNJ, Bartelink H, Bernards R, Giaccone G, Lanschot JJB, Offerhaus GJA. Cancer of the esophagus and gastric cardia: recent advances. Dis Esophagus. 2004;17:10-26.

15. Udagawa H. Chemoradiotherapy: its effectiveness, toxicity, and perspective in the treatment of esophageal cancer. Ann Thorac Cardiovasc Surg. 2009;15:359-61.

Malafaia O. O futuro do câncer de esôfago. Arq Gastroenterol. 2013;50(2)79-80.

DESCRITORES - Neoplasias esofágicas.

*Ex-President of the Brazilian Federation of Gastroenterology - FBG and the Brazilian College of Digestive Surgery - CBCD; Scientific Director of CBCD; Head Professor of Surgery, Federal

University of Paraná and the Evangelical Faculty of Paraná, Curitiba, PR, Brazil. 